\title{
Determinants of fibrotic atrial cardiomyopathy in atrial fibrillation. A multicenter observational study of the RETAC (reseau européen de traîtement d'arrhythmies cardiaques)-group
}

\author{
Björn Müller-Edenborn ${ }^{1,2}$ (1) . Zoraida Moreno-Weidmann ${ }^{1,3}$. Sandrine Venier ${ }^{4}$ Pascale Defaye ${ }^{4}$. Chan-il Park ${ }^{5}$. \\ José Guerra ${ }^{3}$. Concepcion Alonso-Martín ${ }^{3} \cdot$ Victor Bazan $^{3} \cdot$ Xavier Vinolas $^{3}$. Enrique Rodriguez-Font ${ }^{3}$. \\ Bieito Campos Garcia ${ }^{3}$. Serge Boveda ${ }^{6}$. Stéphane Combes ${ }^{6}$. Jean-Paul Albenque ${ }^{6}$. Benoit Guy-Moyat ${ }^{7}$. \\ Dietmar Trenk $^{8} \cdot$ Martin Eichenlaub $^{1} \cdot$ Juan Chen ${ }^{9} \cdot$ Heiko Lehrmann $^{1}$. Franz-Josef Neumann ${ }^{10}$. Thomas Arentz $^{1}$. \\ Amir Jadidi ${ }^{1}$
}

Received: 14 April 2021 / Accepted: 12 November 2021 / Published online: 2 December 2021

(c) The Author(s) 2021

\begin{abstract}
Aims Despite advances in interventional treatment strategies, atrial fibrillation (AF) remains associated with significant morbidity and mortality. Fibrotic atrial myopathy (FAM) is a main factor for adverse outcomes of AF-ablation, but complex to diagnose using current methods. We aimed to derive a scoring system based entirely on easily available clinical parameters to predict FAM and ablation-success in everyday care.

Methods In this multicenter, prospective study, a new risk stratification model termed AF-SCORE was derived in 220 patients undergoing high-density left-atrial(LA) voltage-mapping to quantify FAM. AF-SCORE was validated for FAM in an external mapping-validation cohort $(n=220)$ and for success following pulmonary vein isolation (PVI)-only (without adjunctive left- or right atrial ablations) in an external outcome-validation cohort ( $n=518)$.

Results FAM was rare in patients $<60$ years $(5.4 \%)$, but increased with ageing and affected $40.4 \%(59 / 146)$ of patients $\geq 60$ years. Sex and AF-phenotype had additional predictive value in older patients and remained associated with FAM in multivariate models (odds ratio [OR] 6.194, $p<0.0001$ for $\geq 60$ years; OR 2.863, $p<0.0001$ for female sex; OR $41.309, p<0.0001$ for AF-persistency). Additional clinical or diagnostic variables did not improve the model. AF-SCORE (+1 point for age $\geq 60$ years and additional points for female sex [+1] and AF-persistency [+2]) showed good discrimination to detect FAM (c-statistic 0.792 ) and predicted arrhythmia-freedom following PVI $(74.3 \%, 54.7 \%$ and $45.5 \%$ for
\end{abstract}

Björn Müller-Edenborn and Zoraida Moreno-Weidmann contributed equally.

Björn Müller-Edenborn

bjoern.mueller-edenborn@universitaets-herzzentrum.de

$\triangle$ Amir Jadidi

amir.jadidi@universitaets-herzzentrum.de

1 Department of Cardiology and Angiology II, Section for Electrophysiology, Heart Center, University of Freiburg, Südring 15, 79189 Bad Krozingen, Germany

2 Department of Cardiology, Julius-Hospital, Würzburg, Germany

3 Department of Electrophysiology, Hospital de la Santa Creu i Sant Pau, Universitat Autonoma de Barcelona, CIBERCV, Barcelona, Spain

4 Department of Cardiology, University Hospital Grenoble, Grenoble, France
5 Department of Cardiology, Clinique de la Tour, Geneva, Switzerland

6 Heart Rhythm Management Department, Clinique Pasteur, Toulouse, France

7 Department of Cardiology, University Hospital Limoges, Limoges, France

8 Department of Cardiology and Angiology II, Section for Pharmacology, Heart Center, University of Freiburg, Bad Krozingen, Germany

9 Department of Electrophysiology, University Hospital Mainz, Mainz, Germany

10 Department of Cardiology and Angiology II, Heart Center, University of Freiburg, Bad Krozingen, Germany 
$\mathrm{AF}-\mathrm{SCORE} \leq 2,3$ and 4, respectively, and hazard ratio [HR] 1.994 for AF-SCORE $=3$ and HR 2.866 for AF-SCORE $=4$, $p<0.001)$.

Conclusions Age, sex and AF-phenotype are the main determinants for the development of FAM. A low AF-SCORE $\leq 2$ is found in paroxysmal AF-patients of any age and younger patients with persistent AF irrespective of sex, and associated with favorable outcomes of PVI-only. Freedom from arrhythmia remains unsatisfactory with AF-SCORE $\geq 3$ as found in older patients, particularly females, with persistent AF, and future studies investigating adjunctive atrial ablations to PVI-only should focus on these groups of patients.

\section{Graphical abstract}
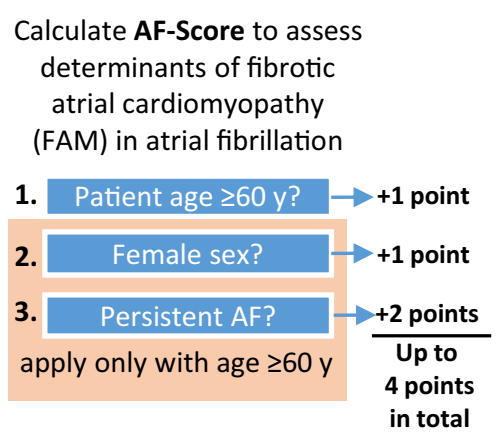

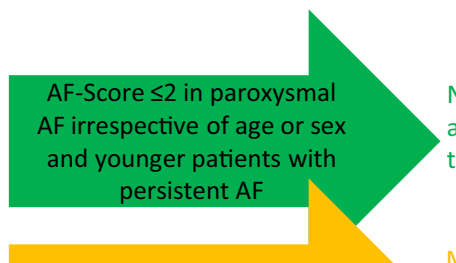

No or only little FAM, low arrhythmia recurrence rates to be expected following PVI

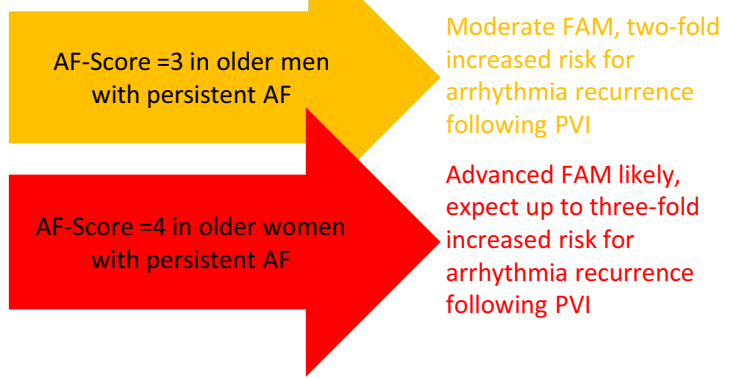

Keywords Atrial fibrillation $\cdot$ Risk stratification $\cdot$ Pulmonary vein isolation $\cdot$ Fibrotic atrial myopathy

\section{Introduction}

Pulmonary vein isolation (PVI) is the mainstay of therapy for the interventional treatment of atrial fibrillation (AF), and arrhythmia freedom can be achieved in up to $80 \%$ of patients $[1,2]$.

In fibrotic atrial myopathy (FAM), areas of atrial fibrosis promote heterogeneous conduction that can itself perpetuate AF even in the absence of pulmonary-vein inputs [3]. As a result, FAM is associated with adverse outcomes following PVI, and arrhythmia recurrences occur in up to $50 \%$ of patients with FAM [4-7].

To select the most appropriate therapeutic strategy in patients with $\mathrm{AF}$, a personalized approach based on the presence or absence of FAM would be desirable. While FAM can be diagnosed non-invasively using contrast-enhanced cardiac magnet resonance imaging or various ECG-parameters, their widespread use for screening-purposes is significantly limited by inherent methodological restrictions and availability.

This is particularly true in the primary care-setting in which the vast majority of patients with AF are taken care for. As a result, several risk stratification models were proposed to identify patients-at-risk for FAM. However, while offering moderate to good diagnostic properties, these models still require diagnostic tests such as echocardiography or blood sampling.

For the current study, we hypothesized that a condensed risk model based entirely on descriptive patient information available through simple medical history taking may yield comparable power to predict FAM and ablation-outcome. In addition, such risk model may allow a better understanding of contemporary trials on AF-ablation, as such descriptive variables are likely available in the published study results.

\section{Methods}

\section{Study design and patient populations}

In this multicenter prospective observational study, consecutive patients undergoing first PVI between January 2016 and October 2019 at the participating sites (University Heart Center Freiburg-Bad Krozingen, Bad Krozingen, Germany; Clinique Pasteur, Toulouse, Centre Hospitalier Universitaire Limoges, Limoges, and Centre Hospitalier Universitaire Grenoble, all in France; Hopital de la Tour, Geneva, Switzerland, and Hopital de Sant Pau, Barcelona, Spain) were screened for inclusion into the determination cohort and underwent high-density endocardial mapping as 
outlined below. An outcome-database of patients who underwent their first PVI at University Heart Center Freiburg-Bad Krozingen and Centre Hospitalier Universitaire Grenoble served as external outcome-validation cohort (Supplemental Fig. 1). Details on ablation and follow-up are described below. Inclusion criteria were symptomatic paroxysmal ( $<7$ days duration) and persistent ( $>7$ days and $<12$ months duration) atrial fibrillation. Exclusion criteria were prior left atrial ablation, presence of left atrial thrombus or contraindication to anticoagulant therapy. Primary endpoints were the derivation of a scoring system to predict left-atrial low voltage-substrate as a surrogate of FAM, and external validation of this score for prediction of FAM. Secondary endpoint was the external validation of this score to predict arrhythmia-freedom following PVI.

\section{High-density mapping of the determination cohort}

Patients in the determination cohort underwent high density left-atrial voltage mapping during sinus rhythm with a minimum of 1200 mapped points per patient [8]. All patients underwent mapping in sinus rhythm prior to any ablation using an endocardial electro-anatomical contact mappingsystem (Carto3, Biosense-Webster, Diamond Bar, CA, US) in combination with a 20-pole circumferential mapping catheter (electrode size: $1 \mathrm{~mm}$; spacing: 2-6-2 mm). Peakto-peak intracardiac bipolar electrograms were recorded at 15 to $250 \mathrm{~Hz}$ and amplified $0.1-0.2 \mathrm{mV} / \mathrm{cm}$ to visualize low-voltage electric activity. For highest accuracy, mapping was performed with respiratory gating and under mechanical ventilation. Low interpolation settings (17 in Carto3-system) were used. Mapping points that were $>5 \mathrm{~mm}$ away from the atrial geometry were excluded. Areas demonstrating potential low voltage when mapped with the 20-pole lasso catheter were reconfirmed using a contact force-enabled mapping catheter with a contact threshold of $>5 \mathrm{~g}$. LVS was defined using a cutoff value for bipolar peak-to-peak voltage in sinus rhythm of $<0.5 \mathrm{mV}$ and further expressed in $\mathrm{cm}^{2}$ of absolute cumulative left-atrial surface area. The pulmonary vein antrae and pulmonary veins that physiologically show voltages $<0.5 \mathrm{mV}$ as well as the mitral valvular area were excluded. For the current study, FAM was defined as LVS exceeding $5 \mathrm{~cm}^{2}$.

\section{Ablation procedure and outcome estimation in the outcome-validation cohort}

All patients of the outcome-validation cohort underwent proximal circumferential pulmonary vein isolation without adjunctive left- or right-atrial ablations using either contact-force enabled radiofrequency-ablation in combination with the Carto3-Mapping system (Biosense Webster) or cryo-balloon-ablation (Medtronic Arctic Front Advance).
The decision per ablation type was at the discretion of the treating physician. Antiarrhythmic drugs, where applicable, were continued for three months following ablation and then stopped. Patients underwent routine ambulatory cardiological examinations including 12-lead ECG at four weeks following ablation. Regular 24 h-Holter-ECGs were scheduled in 6-months-intervals. Patients experiencing symptoms suggestive of arrhythmia recurrence (e.g. palpitations, dyspnea, fatigue) underwent additional cardiological examinations including ECG and symptom-triggered ECG (event recorder and $24 \mathrm{~h}$-Holter ECG). AF or atrial tachycardia lasting for $>30 \mathrm{~s}$ experienced beyond the blanking period of three months were considered as recurrence. The study protocol was approved by the ethic committees of the participating sites.

\section{Statistical analysis}

Statistical analysis was performed using SPSS 25.0 for Windows (IBM Corporation, Armonk, NY) or Graphpad Prism 8 for Windows (Graphpad Software, La Jolla, CA). Data were checked for normal distribution using the Shapiro-Wilk test. Continuous variables were compared between groups using t-test or non-parametric testing or One-way ANOVA with Bonferroni-post hoc correction depending on normality and number of groups. Categorical variables were analyzed using Fisher exact test. Uni- or multivariate logistic regression was used to estimate probability ratios between groups. Unadjusted and adjusted arrhythmia-freedom rates were calculated by cox regression analysis. A two-sided $p \leq 0.05$ was considered significant in all tests.

\section{Results}

\section{Patient characteristics associated with fibrotic atrial myopathy}

In total, 440 patients were included in the determination cohort and underwent high-density left atrial endocardial voltage mapping. Patients of the determination cohort were randomized 1:1 to the derivation cohort or the mappingvalidation cohort ( $n=220$ each, Supplemental Fig. 1). These cohorts did not differ with regard to key patient characteristics (Supplemental Table 1). Factors associated with FAM in the derivation cohort in univariate models were age, female sex, arterial hypertension, persistent AF, left atrial (LA) dilatation and creatinine clearance. In a multivariate logistic model, only age, sex and AF-phenotype remained as independent factors associated with FAM (Table 1). 
Table 1 Uni- and multivariate logistic regression for FAM in the determination cohort

\begin{tabular}{|c|c|c|c|c|c|c|c|}
\hline & \multirow{3}{*}{$\begin{array}{l}\text { All patients } \\
n=220\end{array}$} & \multirow{3}{*}{$\begin{array}{l}\text { No FAM } \\
n=157\end{array}$} & \multirow{3}{*}{$\begin{array}{l}\text { FAM } \\
n=63\end{array}$} & \multicolumn{4}{|l|}{ Regression analysis } \\
\hline & & & & \multicolumn{2}{|l|}{ Unadjusted } & \multicolumn{2}{|l|}{ Adjusted } \\
\hline & & & & OR $(95 \% \mathrm{CI})$ & $p$ & OR $(95 \% \mathrm{CI})$ & $p$ \\
\hline Age (years) & $61.1(11.7)$ & $58.2(12.2)$ & $68.3(6.3)$ & $1.130(1.08-1.18)$ & $<0.0001$ & & \\
\hline Age $>60$ years & $146(66.4)$ & $87(55.4)$ & $59(93.7)$ & $11.868(4.11-34.27)$ & $<0.0001$ & $6.194(1.94-19.8)$ & 0.002 \\
\hline Female sex & $60(27.3)$ & $34(21.7)$ & $26(41.3)$ & $2.542(1.36-4.77)$ & 0.004 & $2.863(1.28-6.4)$ & 0.011 \\
\hline Body mass index (kg/sqm) & $28.0(4.4)$ & $27.9(4.2)$ & $28.2(4.7)$ & $1.018(0.95-1.09)$ & 0.598 & & \\
\hline Arterial hypertension & $139(63.2)$ & $89(56.7)$ & $50(79.4)$ & $2.939(1.48-5.84)$ & 0.002 & $1.147(0.49-2.70)$ & 0.753 \\
\hline Diabetes mellitus & $19(8.6)$ & $11(7.0)$ & $8(12.7)$ & $1.931(0.74-5.05)$ & 0.180 & & \\
\hline Coronary artery disease & $37(16.8)$ & $23(14.6)$ & $14(22.2)$ & $1.665(0.79-3.49)$ & 0.177 & & \\
\hline Persistent atrial fibrillation & $144(65.5)$ & $82(52.2)$ & $62(98.4)$ & $56.71(7.7-419.1)$ & $<0.0001$ & $41.309(5.34-319.48)$ & $<0.0001$ \\
\hline LV dysfunction $(\mathrm{LVEF}<45 \%)$ & $17(7.7)$ & $10(6.4)$ & $7(11.1)$ & $1.837(0.67-5.06)$ & 0.239 & & \\
\hline LVEDD (mm) & $51.1(4.4)$ & $51.2(4.5)$ & $50.9(4.3)$ & $0.981(0.92-1.05)$ & 0.564 & & \\
\hline LA dilatation $(>40 \mathrm{~mm})$ & $173(78.6)$ & $115(73.2)$ & $58(92.1)$ & $4.237(1.59-11.28)$ & 0.004 & $2.221(0.69-7.15)$ & 0.181 \\
\hline $\begin{array}{l}\text { Creatinine clearance }(\mathrm{ml} / \mathrm{min} / 1.73 \\
\text { sqm) }\end{array}$ & $78.3(15.1)$ & $80.1(14.7)$ & $73.8(15.2)$ & $0.972(0.95-0.99)$ & 0.006 & $0.997(0.97-1.02)$ & 0.789 \\
\hline Cumulative area $<0.5 \mathrm{mV}(\mathrm{sqcm})$ & $6.1(12.0)$ & $0.8(1.3)$ & $19.2(16.0)$ & $\mathrm{n} / \mathrm{a}$ & & & \\
\hline cumulative area $<1.0 \mathrm{mV}(\mathrm{sqcm})$ & $15.2(21.1)$ & $5.0(6.2)$ & $40.5(23.5)$ & $\mathrm{n} / \mathrm{a}$ & & & \\
\hline
\end{tabular}

FAM, fibrotic atrial myopathy (prespecified as $\geq 5 \mathrm{~cm}^{2}$ with bipolar voltage $<0.5 \mathrm{mV}$ ); LA, left atrial; LV, left ventricle; LVEDD, left-ventricular end-diastolic diameter

Values are given as mean \pm standard deviation or $n(\%)$
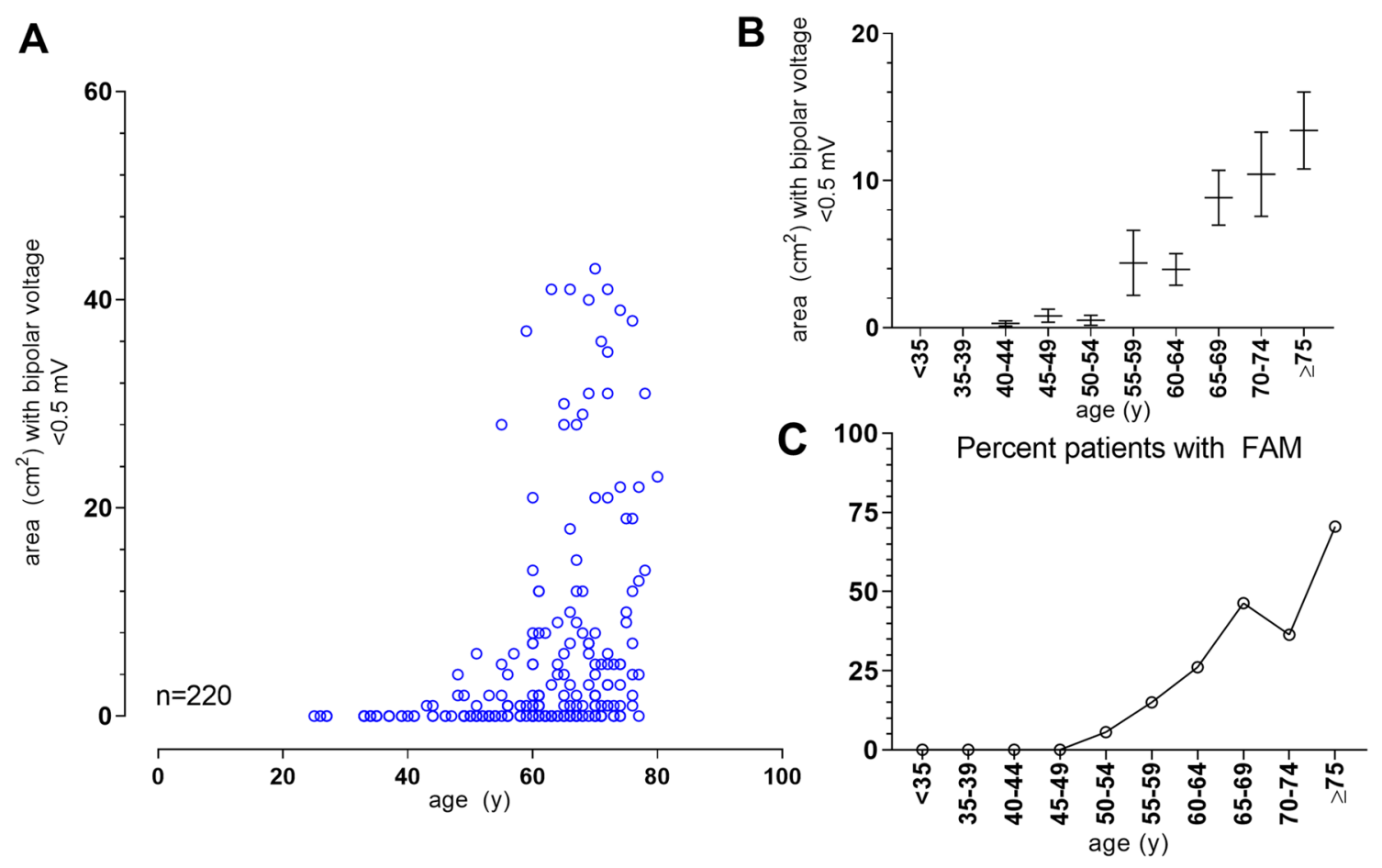

Fig. 1 Age-related development of fibrotic atrial myopathy. Shown is the cumulative area with bipolar voltages $<0.5 \mathrm{mV}$ per patient vs. patient age in the derivation cohort (A). Panel (B) gives the mean and standard error of cumulative areas with voltages $<0.5 \mathrm{mV}$ in age-cat- egories spanning 5 years each. The percentage of patients within each given age group meeting the prespecified criterion for FAM $\left(\geq 5 \mathrm{~cm}^{2}\right.$ low-voltage areas at $<0.5 \mathrm{mV}$ ) is shown in (C). FAM, fibrotic atrial myopathy 


\section{Age-related development of fibrotic atrial myopathy}

For the derivation cohort, the cumulative left-atrial area demonstrating bipolar voltages $<0.5 \mathrm{mV}$ in relation to the patient's age is given in Fig. 1. Patients who met the prespecified criterion for diagnosis of FAM $\left(\geq 5 \mathrm{~cm}^{2}\right.$ lowvoltage areas at $<0.5 \mathrm{mV}$ ) had on average $19.2 \pm 15.9 \mathrm{~cm}^{2}$ of cumulative low-voltage areas, compared to $0.8 \pm 1.3$ $\mathrm{cm}^{2}$ in patients who did not. Extensive low-voltage areas were primarily found in patients aged 60 years or older $\left(8.4 \pm 13.6 \mathrm{~cm}^{2}\right.$ vs. $1.4 \pm 5.4 \mathrm{~cm}^{2}$ in patients younger 60 years, $p<0.0001)$. The criterion for FAM $\left(\geq 5 \mathrm{~cm}^{2}\right.$ lowvoltage areas at $<0.5 \mathrm{mV}$ ) was met in 59 of 146 patients $(40.4 \%)$ aged 60 years or older, compared to only 4 of 70 (5.4\%) in younger patients $(p<0.0001)$. The percentage of patients in a given age group with FAM increased steadily from $0 \%(0 / 38)$ in patients younger 44 years to $36 \%(31 / 87)$ in patients aged 60-69 years and reached 70\% (12/17) in those aged 75 years or older (Fig. 1).

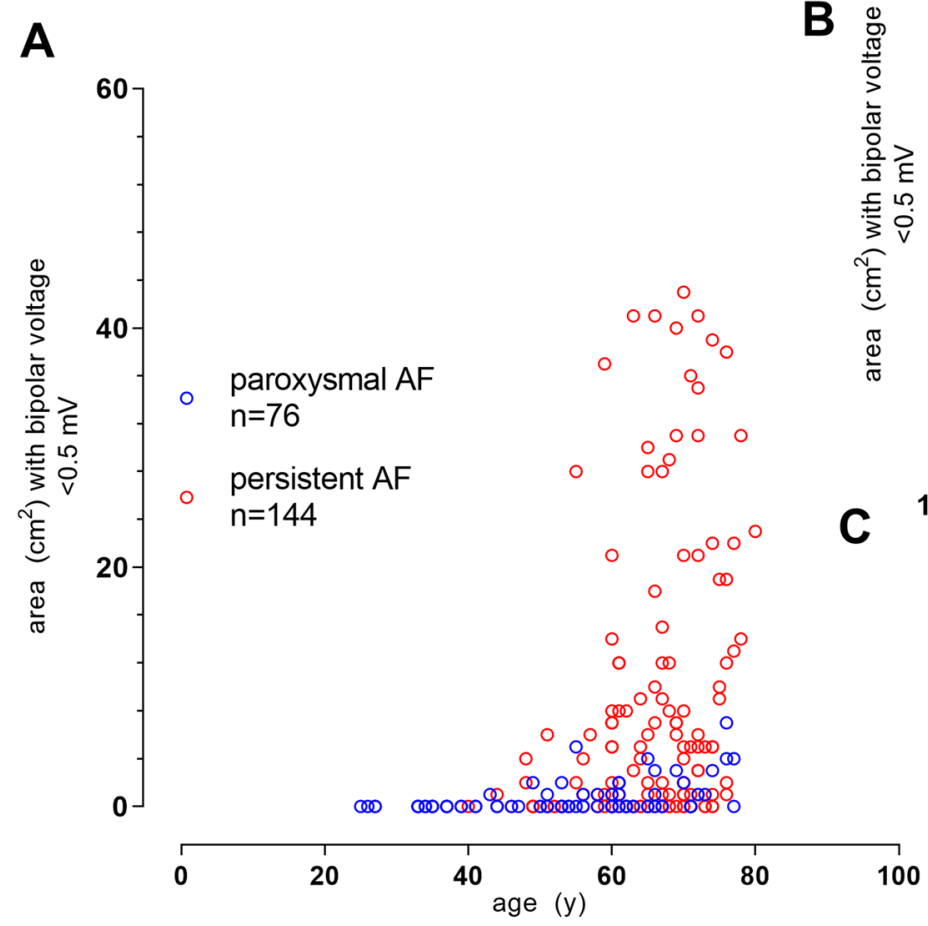

Fig. 2 Clinical AF-phenotype and age-dependency of fibrotic atrial myopathy. Shown is the cumulative area with bipolar voltages $<0.5 \mathrm{mV}$ per patient vs. patient age in the derivation cohort, grouped for paroxysmal AF (blue) or persistent AF (red, Panel A). Panel (B) gives the mean and standard error of cumulative areas with voltages $<0.5 \mathrm{mV}$ in age-categories spanning 5 years each separated

\section{Impact of clinical AF-phenotype on presence of fibrotic atrial myopathy}

Persistent AF with the longest recorded episode of AF exceeding 7 days was present in $42 \%$ (31/74) of younger and $77 \%(113 / 146)$ of patients aged 60 years or older $(p<0.0001)$. The presence of FAM increased incrementally with age in patients with persistent $\mathrm{AF}$, reaching up to $84 \%$ in those aged 75 years or older (Fig. 2). Younger patients ( $<60$ years) rarely had FAM despite a clinical phenotype of persistent $\mathrm{AF}$ (4/31, 12.9\%).

This observation was also made regarding the absolute extent of low-voltage areas, which were higher in persistent compared to paroxysmal AF only in patients older than 60 years $\left(10.5 \pm 14.8 \mathrm{~cm}^{2}\right.$ vs. $1.2 \pm 1.7 \mathrm{~cm}^{2}$ and $3.0 \pm 8.1$ $\mathrm{cm}^{2}$ vs. $0.3 \pm 0.8 \mathrm{~cm}^{2}$ for persistent vs. paroxysmal $\mathrm{AF}$ in patients older and younger than 60 years, respectively; Supplemental Fig. 2).

Irrespective of age, FAM was rare in patients with paroxysmal AF and occurred in 1.3\% (1/76) of cases only. The negative predictive value of a paroxysmal AF-phenotype for the absence of FAM was 100\% in younger and $96.9 \%$ in patients aged 60 years and older. Irrespective of age,

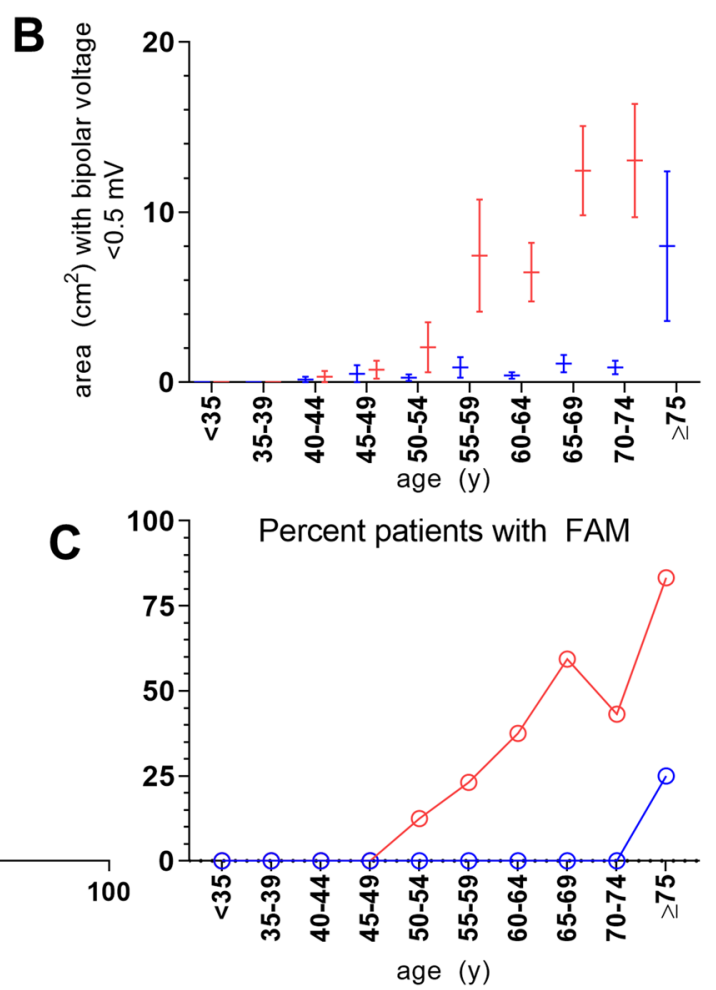

for paroxysmal AF (blue) or persistent AF (red). The percentage of patients within a given age group meeting the prespecified criterion for FAM ( $\geq 5 \mathrm{~cm}^{2}$ low-voltage areas at $\left.<0.5 \mathrm{mV}\right)$ in the respective groups is shown in (C). AF, atrial fibrillation; FAM, fibrotic atrial myopathy 
AF-persistency was highly sensitive, yet not specific, for FAM (Supplemental Fig. 2B).

\section{Female Sex and fibrotic atrial myopathy}

While the majority of study patients were male $(160 / 220$, $72.7 \%$ ), women had FAM more often and more severe than men $(26 / 60$ females with FAM [43.3\%] vs $37 / 160$ males [23.1\%], $p<0.0001$; and low-voltage areas $<0.5 \mathrm{mV}$ of $10.9 \pm 17.2 \mathrm{~cm}^{2}$ in females vs. $4.2 \pm 8.7 \mathrm{~cm}^{2}$ in males, $p<0.0001$, Supplemental Fig. 3). The higher overall prevalence of FAM in females was driven by older women with persistent AF (Figs. 3 and $4 ; 18.1 \pm 19.5 \mathrm{~cm}^{2}$ in females with persistent AF aged $\geq 60$ years vs. $7.1 \pm 10.5$ $\mathrm{cm}^{2}$ in males with persistent $\mathrm{AF}$ aged $\geq 60$ years, $p<0.0001)$. In younger patients, sex did not affect the extent of FAM (Supplemental Fig. 3, $p=0.389$ ).

\section{Clinical risk stratification for fibrotic atrial myopathy and outcome-prediction in pulmonary vein isolation: the AF-SCORE}

Based on multivariate logistic models (Table 1), age, sex and AF-phenotype were incorporated in a simple risk
Fig. 3 Impact of sex on fibrotic atrial myopathy. The cumulative area with voltages $<0.5 \mathrm{mV}$ in individual patients in the derivation cohort vs. patient age and sex (females in red, males in blue) is shown in the large Panel. The inlay demonstrates the percentage of females and males meeting the prespecified criterion for FAM $\left(\geq 5 \mathrm{~cm}^{2}\right.$ lowvoltage areas at $<0.5 \mathrm{mV}$ )

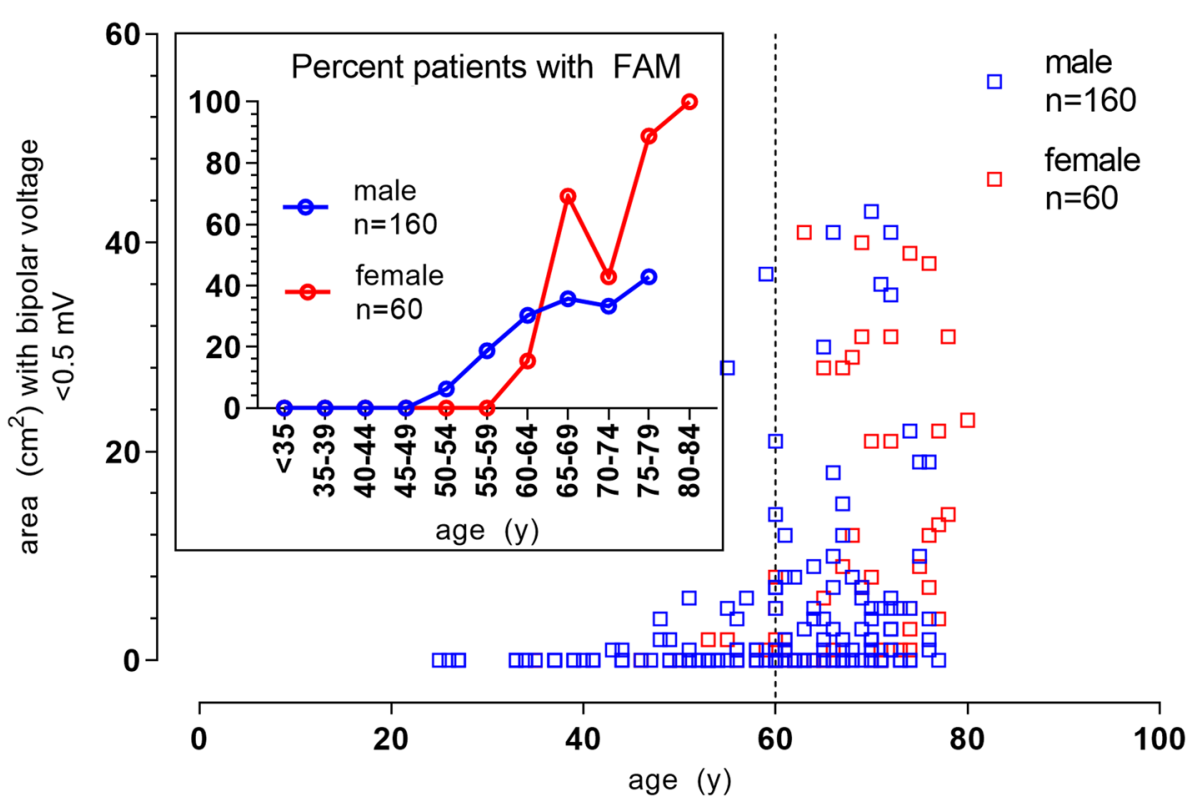

Fig. 4 Sex and AF-phenotype determine fibrotic atrial myopathy in advanced age. The large panel shows the distribution of areas with bipolar voltages $<0.5 \mathrm{mV}$ in the derivation cohort in women (red), men (blue) with paroxysmal (circles) and persistent $\mathrm{AF}$ (triangles). The small panel compares the extent of low-voltage areas between sexes and AF-phenotype restricted to patients aged $\geq 60$ years. AF, atrial fibrillation

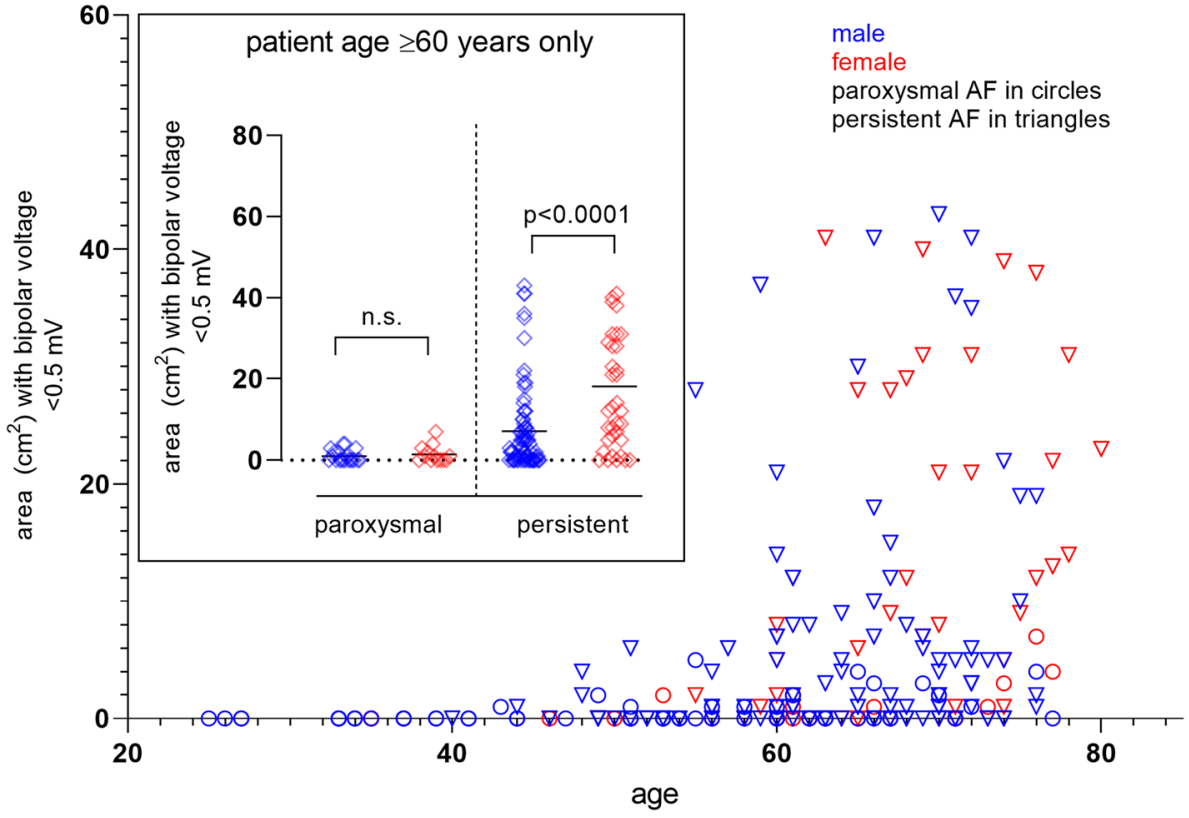




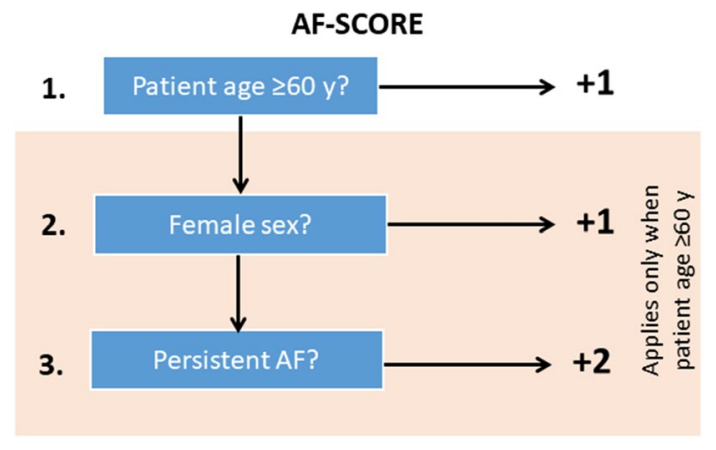

Fig. 5 Determination of AF-SCORE. AF-SCORE is determined using patient age, sex, and clinical phenotype of AF. Patient age $\geq 60$ years constitutes 1 point. Female sex ( +1 point) and persistency of $\mathrm{AF}(+2$ points) are added in patients aged $\geq 60$ years. $\mathrm{AF}$, atrial fibrillation

stratification scheme called AF-SCORE that ranges from 0 to 4 points (Fig. 5). One point is given when a patient's age is $\geq 60$ years, with additional points in older patients for female sex (1 point) and persistent AF-phenotype (2 points).

Forty-nine percent of patients in the mapping-validation cohort had an AF-SCORE $\leq 2$. For "rule-out" of significant LVS, this threshold yielded a sensitivity of $92.0 \%$ and a negative-predictive value of $95.3 \%$. A pathological threshold for AF-SCORE of $\geq 3$ selected $51.4 \%$ of patients in the validation cohort and yielded a specificity of $64.9 \%$ and a positive-predictive value of $51.3 \%$ to "rule-in" significant LVS. Increasing the pathological threshold for AF-SCORE to 4 selected $15.9 \%$ of study patients and yielded a specificity of $93.6 \%$ and an increased positive-predictive value of $71.4 \%$ for "rule-in".

Application of AF-SCORE to the mapping-validation cohort is shown in Fig. 6A-C. Receiver-operating-curveanalysis yielded an area-under-the-curve (c-statistic) for presence of FAM of 0.792 (Fig. 6A). An AF-SCORE of $\leq 2$ is related to absence of, respective very minor FAM, which increases with an incremental AF-SCORE (Fig. 6B; $1.7 \pm 3.9 \mathrm{~cm}^{2}$ for an AF-SCORE of $\leq 2,9.6 \pm 13.5 \mathrm{~cm}^{2}$ for an AF-SCORE of 3 , and $24.8 \pm 28.2 \mathrm{~cm}^{2}$ for an AF-SCORE of $4, p<0.0001)$. Accordingly, the proportion of patients with FAM ( $>5 \mathrm{~cm}^{2}$ at $<0.5 \mathrm{mV}$ ) increased incrementally from AF-SCORE $\leq 2$ to AF-SCORE of 4 (Fig. 6C).

AF-SCORE as determined above was next applied on the outcome-validation cohort comprising 518 patients in total who underwent proximal-circumferential pulmonary vein isolation for symptomatic AF. Baseline characteristics of these patients with regard to outcome are given in supplemental table 2 and with regard to AF-SCORE in supplemental table 3. Patients with recurrence were older, predominantly female, had more often persistent AF, were more frequently ablated using radiofrequency than cryo-ablation and had a higher AF-SCORE. During a mean follow-up of $421 \pm 208$ days, 172 patients experienced AF-recurrence.

Patients with AF-SCORE of 3 or 4 demonstrated significantly more recurrences as opposed to patients with AF-SCORE 2 or lower (unadjusted hazard ratio 1.994 [95\% confidence interval 1.44-2.77] for AF-SCORE 3 and HR 2.866 [95\% CI 1.89-4.37] for AF-SCORE 4 compared to AF-SCORE $\leq 2$; Fig. 6D). These differences persisted when the analysis was adjusted for type of ablation (HR 1.595 [95\% CI 1.1-2.34] for AF-SCORE 3 and HR 2.231 [95\% CI 1.39-3.59] for AF-SCORE 4 compared to AF-SCORE $\leq 2$ ) or pre-procedural antiarrhythmic medications (HR 2.081 [95\% CI 1.46-2.96] for AF-SCORE 3 and HR 3.443 [95\% CI 2.22-5.33] for AF-SCORE 4 compared to AF-SCORE $\leq 2$. Arrhythmia freedom rates were $74.3 \%(\mathrm{AF}-\mathrm{SCORE} \leq 2$ ), $54.7 \%$ (AF-SCORE 3) and 45.5\% (AF-SCORE 4).

\section{Discussion}

Our study reveals three major findings concerning the development of fibrotic atrial myopathy (FAM) in atrial fibrillation: first, age is the main factor in the development of FAM, and FAM is rare in those younger than 60 years. Second, sex and clinical phenotype of atrial fibrillation (AF) are important determinants with advanced age, but can de neglected in young patients. And third, incorporation of patient's age, sex and AF-phenotype into AF-SCORE yields a simple, widely applicable tool to approximate the individual risk for FAM and to estimate the success rate of pulmonary vein isolation in both paroxysmal and persistent AF.

\section{Clinical risk scores for fibrotic atrial myopathy}

Fibrotic remodeling of previously healthy atrial myocardium is associated with slow-conduction sites that serve as arrhythmogenic substrate and contribute to the perpetuation of $\mathrm{AF}$ even in the absence of pulmonary vein-inputs [3, 5 , 9]. Fibrotic regions can be identified either as areas with reduced local voltage by endocardial contact mapping or by delayed Gadolinium-enhanced areas using MRI [10]. No matter the diagnostic modality, advanced fibrotic remodeling was demonstrated to be a main factor determining the longterm success of PVI $[5,6,8,11,12]$. In general practice, the use of these tools is, however, limited by prohibitive costs (MRI), invasiveness (contact mapping) and/or required expertise (electrocardiographic markers) [8, 12].

These limitations led to the development of several scoring systems that aim at stratifying patients for the presence of fibrotic atrial remodeling such as the DR-FLASH and APPLE-score [13, 14]. With an area-under-the-curve of 0.711 (APPLE-score) and 0.797 (DR-FLASH), they offer moderate to good discriminative properties for the diagnosis 

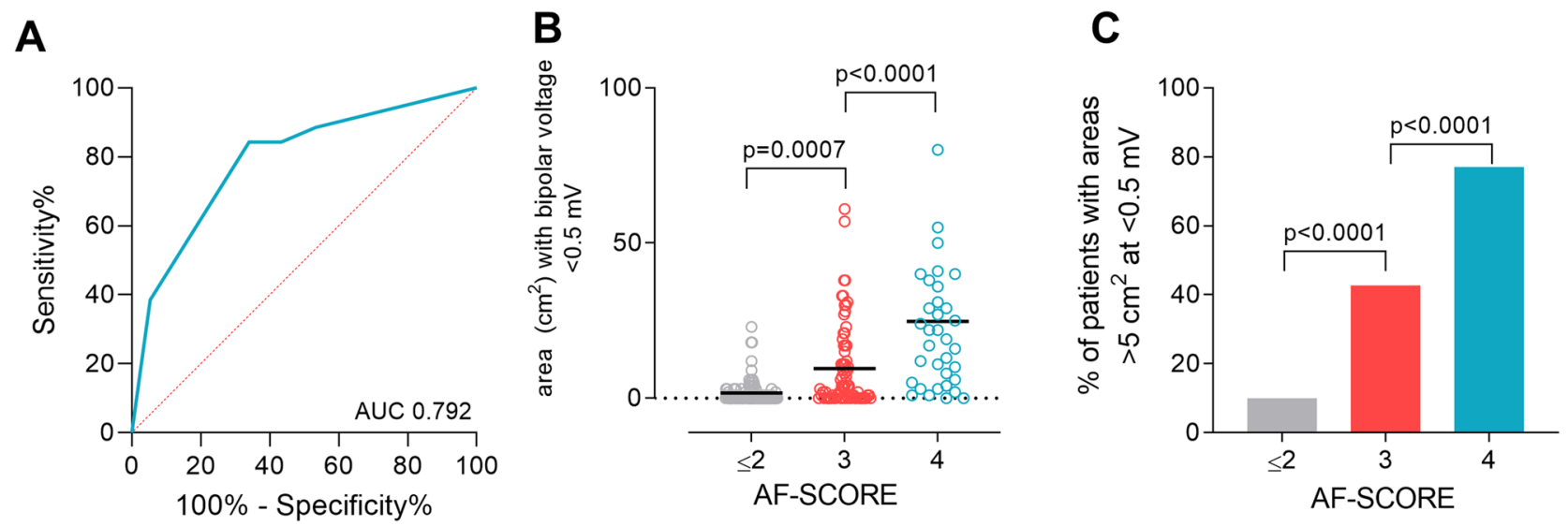

Mapping-Validation Cohort $\mathrm{N}=\mathbf{2 2 0}$

D Outcome-Validation Cohort $\mathrm{N}=518$

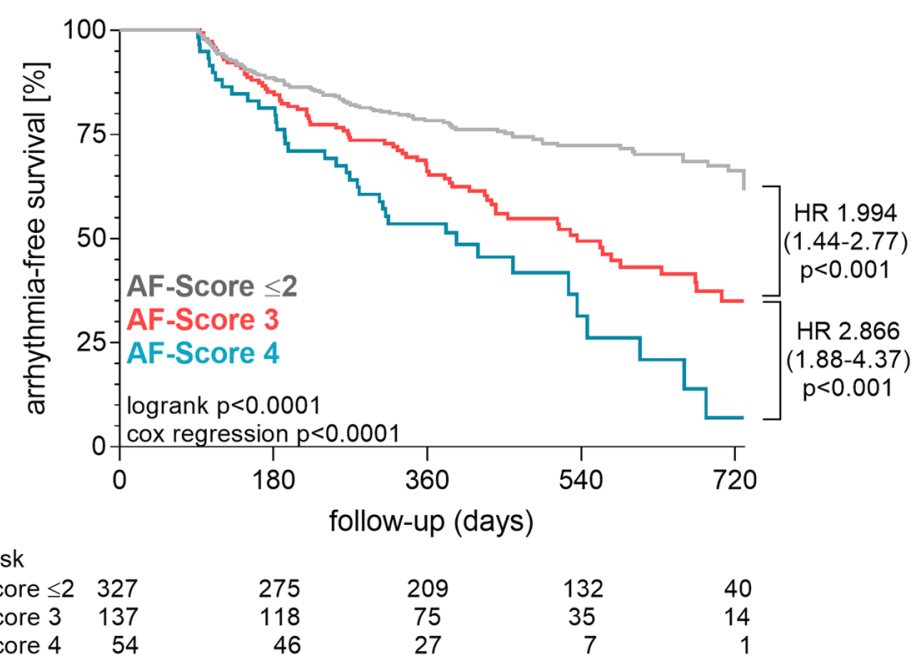

Fig. 6 Diagnostic and prognostic properties of AF-SCORE. Receiveroperating-curve analysis of AF-SCORE for presence of FAM $(\geq 5$ $\mathrm{cm}^{2}$ low-voltage areas at $<0.5 \mathrm{mV}$ ) in the derivation cohort is given in (A). The absolute cumulative area with voltages $<0.5 \mathrm{mV}$ with regard to AF-SCORE is given in $(\mathbf{B})$. The percentage of patients with FAM, defined as cumulative areas with bipolar voltages $<0.5 \mathrm{mV}$

of atrial low-voltage substrate, but require echocardiographic or laboratory parameters such as left atrial diameter, leftventricular ejection fraction or renal function in addition to routine patients' clinical characteristics. In contrast, AFSCORE as described in the current study relies solely on patient characteristics that are part of every AF-patient's medical history: age, sex, and whether AF is paroxysmal or persistent. This greatly condensed clinical risk stratification model yields diagnostic properties (AUC 0.792) that are on par with the abovementioned scores and others, and AFSCORE proved equally efficient to predict PVI-outcome. of $>5 \mathrm{~cm}^{2}$ in relation to AF-SCORE, is shown in (C). Kaplan-Meierestimates of arrhythmia-free survival following pulmonary vein isolation without additional left- or right-atrial ablations depending on AF-SCORE in the outcome-validation cohort is shown in (D). AUC, area under curve, HR, hazard ratio

\section{Atrial fibrosis in the aging heart}

The current study underlines that age is a major determinant for the development of extra-pulmonary vein arrhythmogenic fibrotic substrate in the left atrium. This finding spreads new light on the interpretation of established clinical trials on AF-ablation, particularly on those that focus on persistent forms of AF: the STAR-AF II-trial compared PVI-only to complex fractionated atrial electrogram (CFAE)-ablation or linear ablation in addition to PVI, and found no benefit of adjunctive left atrial ablations [15]. This trial enrolled younger patients at a mean age of 58-61 years. Similar findings were obtained in the CHASE-AF trial, 
which compared a stepwise approach including widespread bi-atrial ablations ("full-defragmentation") to PVI-only in persistent-AF patients [16]. At a mean age of 61 in the "full-defrag"-group, no additional benefit to PVI-only was observed.

Taking the current study's data into account, a large proportion of patients in STAR-AF II and CHASE-AF likely had no relevant left-atrial arrhythmogenic fibrotic substrate, but rather isolated pulmonary vein-dependent $\mathrm{AF}$ - no matter of the clinical phenotype of persistent AF.

As a result, ablation strategies aiming at non-pulmonary vein-sources of $A F$ such as linear ablations and ablation of high-voltage complex-fractionated atrial electrograms were in retrospect unlikely to show an additional benefit in these trials [15].

\section{Atrial fibrosis in the female heart}

In patients aged 60 years or older, the current study found extensive non-pulmonary vein fibrotic substrate predominantly in females. However, women of any age are widely underrepresented in key clinical trials on AF-ablation: STAR-AF II and CHASE-AF included approximately $80 \%$ of male participants, and $87 \%$ of patients randomized to ablation in CASTLE-AF (comparing ablation to conservative medical therapy in patients with atrial fibrillation and heart failure) were males [17]. Even so, women in CASTLEAF had no discernable benefit of catheter ablation in contrast to men in subgroup analysis.

Female sex seems disadvantageous with regard to fibrotic myocardial remodeling in at least two aspects. First, women, but not men, develop myocardial fibrosis with age also in the absence of cardiovascular risk factors [18]. And second, in specific cardiovascular disease such as valvular cardiomyopathy and despite a more beneficial overall cardiovascular risk profile, women have a higher burden of myocardial fibrosis than men [19].

\section{PV-dependent and non-PV-substrate-dependent persistent atrial fibrillation in contemporary clinical trials}

Guidelines define persistent AF as individual AF-episodes that vary in duration from seven days to less than 12 months. The current study illustrates that this population is not homogeneous, but composed of two very different groups of patients: younger patients, in whom $\mathrm{AF}$ is largely PV-dependent, no matter the clinical AF-phenotype or sex, and older patients, particularly women (74\% of females $\geq 60$ years with persistent AF vs. $55 \%$ of males had FAM), who likely have fibrotic atrial substrate resulting in a "non-PV-substrate-dependent" form of persistent AF.
Unfortunately, many key clinical trials on AF-ablation in general and ablation of persistent AF in particular were conducted in patient populations consisting of relatively young, male, and presumably PV-dependent AF-patients (Supplemental Fig. 4). Future studies investigating adjunctive leftatrial ablations in addition to PVI should therefore focus on patients with the highest likelihood of non-PV-substratedependency: senior patients and women with persistent AF.

\section{Limitations}

The current study uses endocardial contact mapping to quantify FAM, which is routinely used for this purpose and can be considered the clinical standard. However, atrial histology would constitute the gold standard to quantify the fibrotic remodeling in FAM, but is unavailable in clinical practice.

Also, AF-SCORE was determined in a derivation cohort (supplemental Fig. 1) and then validated for FAM-extent and PVI-outcome in two separate, external validation cohorts (the so-called mapping-validation cohort and outcome-validation cohort; Fig. 6A-C and D, respectively). As not all patients in the outcome-validation cohort underwent high-density mapping, a direct link of FAM-extent to PVI-outcome can however not be extrapolated from the current study's data.

\section{Conclusions}

AF-SCORE stratifies atrial fibrillation-patients for FAM and risk for arrhythmia recurrences with clinically relevant diagnostic reliability. With expected arrhythmia freedom rates within two years from ablation approaching $75 \%$, paroxysmal AF-patients of any age and younger patients with persistent $\mathrm{AF}$ are, irrespective of sex, promising candidates for PVI-only-techniques using either radiofrequency-ablation or single shot-cryo ablation. Challenges do remain in older patients with persistent $\mathrm{AF}$, and particularly older women, in whom arrhythmia freedom can be achieved in only in half of patients using current PVI-only approaches.

Supplementary Information The online version contains supplementary material available at https://doi.org/10.1007/s00392-021-01973-1.

Author contributions BME and ZMW designed the study, recruited patients, analyzed the data and drafted the manuscript. SV, PD, CP, JG, CAM, VB, XV, ERF, BCG, SB, SC, JPA, ME, JC, HL and BGM recruited patients and acquired data. DT and FJN analyzed the data and drafted the manuscript. TA and AJ designed the study, analyzed the data and drafted the manuscript. 
Funding Open Access funding enabled and organized by Projekt DEAL. From internal sources.

Availability of data and materials Data will be made available upon reasonable request.

\section{Declarations}

Conflicts of interest The authors report no conflicts of interest in relation to this manuscript.

Ethics approval and consent to participate/publication The study protocol was approved by the ethic committees of the participating sites and patients consented for participation and publication.

Open Access This article is licensed under a Creative Commons Attribution 4.0 International License, which permits use, sharing, adaptation, distribution and reproduction in any medium or format, as long as you give appropriate credit to the original author(s) and the source, provide a link to the Creative Commons licence, and indicate if changes were made. The images or other third party material in this article are included in the article's Creative Commons licence, unless indicated otherwise in a credit line to the material. If material is not included in the article's Creative Commons licence and your intended use is not permitted by statutory regulation or exceeds the permitted use, you will need to obtain permission directly from the copyright holder. To view a copy of this licence, visit http://creativecommons.org/licenses/by/4.0/.

\section{References}

1. Phlips T, Taghji P, El Haddad $\mathrm{M}$ et al (2018) Improving procedural and one-year outcome after contact force-guided pulmonary vein isolation: the role of interlesion distance, ablation index, and contact force variability in the 'CLOSE'-protocol. Europace 20:f419-f427. https://doi.org/10.1093/europace/eux376

2. Kuck K-H, Brugada J, Fürnkranz A et al (2016) Cryoballoon or radiofrequency ablation for paroxysmal atrial fibrillation. $\mathrm{N}$ Engl J Med 374:2235-2245. https://doi.org/10.1056/NEJMoa1602014

3. Jadidi A, Nothst M, Chen J et al (2020) Specific electrogram characteristics identify the extra-pulmonary vein arrhythmogenic sources of persistent atrial fibrillation-characterization of the arrhythmogenic electrogram patterns during atrial fibrillation and sinus rhythm. Nat Sci Rep 10:1-12. https://doi.org/10.1038/ s41598-020-65564-2

4. Verma A, Mantovan R, Macle L et al (2010) Substrate and trigger ablation for reduction of atrial fibrillation (STAR AF): a randomized, multicentre, international trial. Eur Heart J 31:13441356. https://doi.org/10.1093/eurheartj/ehq041

5. Jadidi AS, Lehrmann H, Keyl C et al (2016) Ablation of persistent atrial fibrillation targeting low-voltage areas with selective activation characteristics. Circ Arrhythmia Electrophysiol 9:e002962. https://doi.org/10.1161/CIRCEP.115.002962

6. Rolf S, Kircher S, Arya A et al (2014) Tailored atrial substrate modification based on low-voltage areas in catheter ablation of atrial fibrillation. Circ Arrhythmia Electrophysiol 7:825-833. https://doi.org/10.1161/CIRCEP.113.001251

7. Platonov PG, Mitrofanova LB, Orshanskaya V et al (2011) Structural abnormalities in atrial walls are associated with presence and persistency of atrial fibrillation but not with age. J Am Coll Cardiol 58:2225-2232. https://doi.org/10.1016/j.jacc.2011.05.061

8. Jadidi A, Müller-Edenborn B, Chen J et al (2018) The duration of the amplified sinus-P-wave identifies presence of left atrial low voltage substrate and predicts outcome after pulmonary vein isolation in patients with persistent atrial fibrillation. JACC Clin Electrophysiol 4:531-543. https://doi.org/10.1016/j.jacep.2017. 12.001

9. Haissaguerre M, Shah AJ, Cochet $\mathrm{H}$ et al (2016) Intermittent drivers anchoring to structural heterogeneities as a major pathophysiological mechanism of human persistent atrial fibrillation. J Physiol 594:2387-2398. https://doi.org/10.1113/JP270617

10. Oakes RS, Badger TJ, Kholmovski EG et al (2009) Detection and quantification of left atrial structural remodeling with delayedenhancement magnetic resonance imaging in patients with atrial fibrillation. Circulation 119:1758-1767. https://doi.org/10.1161/ CIRCULATIONAHA.108.811877

11. Marrouche NF, Wilber D, Hindricks G et al (2014) Association of atrial tissue fibrosis identified by delayed enhancement MRI and atrial fibrillation catheter ablation: the DECAAF study. JAMA 311:498-506. https://doi.org/10.1001/jama.2014.3

12. Müller-Edenborn B, Chen J, Didenko M et al (2019) Amplified sinus-P-wave reveals localization and extent of left atrial lowvoltage substrate: implications for arrhythmia freedom following pulmonary vein isolation. EP Eur 2019:1-10. https://doi.org/10. 1093/europace/euz297

13. Kornej J, Büttner P, Sommer P et al (2019) Prediction of electro-anatomical substrate using APPLE score and biomarkers. Europace 21:54-59. https://doi.org/10.1093/europace/euy 120

14. Kosiuk J, Dinov B, Kornej J et al (2015) Prospective, multicenter validation of a clinical risk score for left atrial arrhythmogenic substrate based on voltage analysis: DR-FLASH score. Hear Rhythm 12:2207-2212. https://doi.org/10.1016/j.hrthm.2015. 07.003

15. Verma A, Jiang C, Betts TR et al (2015) Approaches to catheter ablation for persistent atrial fibrillation. N Engl J Med 372:18121822. https://doi.org/10.1056/NEJMoa1408288

16. Vogler J, Willems S, Sultan A et al (2015) Pulmonary vein isolation versus defragmentation the CHASE-AF clinical trial. J Am Coll Cardiol 66:2743-2752. https://doi.org/10.1016/j.jacc.2015. 09.088

17. Marrouche NF, Brachmann J, Andresen D et al (2018) Catheter ablation for atrial fibrillation with heart failure. N Engl J Med 378:417-427. https://doi.org/10.1056/NEJMoa1707855

18. Liu CY, Liu YC, Wu C et al (2013) Evaluation of age-related interstitial myocardial fibrosis with cardiac magnetic resonance contrast-enhanced T1 mapping: MESA (Multi-Ethnic Study of Atherosclerosis). J Am Coll Cardiol 62:1280-1287. https://doi. org/10.1016/j.jacc.2013.05.078

19. Singh A, Musa TA, Treibel TA et al (2019) Sex differences in left ventricular remodelling, myocardial fibrosis and mortality after aortic valve replacement. Heart 105:1818-1824. https://doi.org/ 10.1136/heartjnl-2019-314987 